Acta Crystallographica Section F

Structural Biology

and Crystallization

Communications

ISSN 1744-3091

\section{Takuya Miyakawa, ${ }^{a}$ Yoriko Sawano, ${ }^{a}$ Ken-ichi Miyazono, ${ }^{a}$ Ken-ichi Hatano ${ }^{b}$ and Masaru Tanokura $^{\mathrm{a} *}$}

a Department of Applied Biochemical Chemistry, Graduate School of Agricultural and Life Sciences, University of Tokyo, Bunkyo-ku, Tokyo 113-8657, Japan, and ${ }^{\mathbf{b}}$ Department of Chemistry and Chemical Biology, Faculty of Engineering, Gunma University, Kiryu, Gunma 376-8515, Japan

Correspondence e-mail: amtanok@mail.ecc.u-tokyo.ac.jp

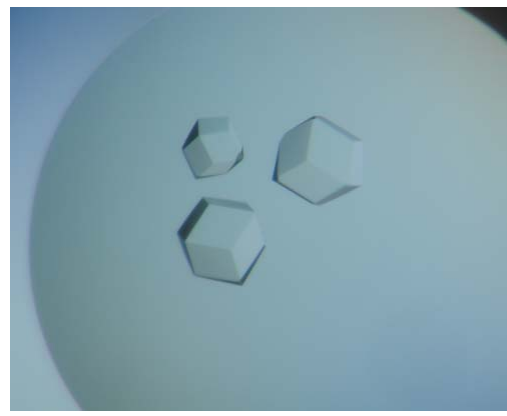

C 2007 International Union of Crystallography All rights reserved

\section{Crystallization and preliminary X-ray analysis of ginkbilobin-2 from Ginkgo biloba seeds: a novel antifungal protein with homology to the extracellular domain of plant cysteine-rich receptor-like kinases}

The antifungal protein ginkbilobin-2 (Gnk2) from Ginkgo biloba seeds does not show homology to other pathogenesis-related proteins, but does show homology to the extracellular domain of plant cysteine-rich receptor-like kinases. Native Gnk2 purified from ginkgo nuts and the selenomethionine derivative of recombinant Gnk2 (SeMet-rGnk2) were crystallized by the sitting-drop vapourdiffusion method using different precipitants. X-ray diffraction data were collected from Gnk2 at $2.38 \AA$ resolution and from SeMet-rGnk2 at $2.79 \AA$ resolution using a synchrotron-radiation source. The crystals of both proteins belonged to the primitive cubic space group $P 2_{1} 3$, with unit-cell parameters $a=b=c=143.2 \AA$.

\section{Introduction}

Plants have evolved a variety of potent defence mechanisms, including the synthesis of secondary metabolites and antimicrobial proteins (Wallace, 2004; Selitrennikoff, 2001), since they have no immune system against pathogenic fungi and bacteria. In the plant life cycle, the seed-germination period is especially vulnerable to pathogen attack because rupture of the seed coat could allow pathogens to invade the seed-storage tissues. Therefore, many plant seeds contain antifungal and antibacterial proteins to protect the nutritious tissues from invaders. Recently, we found that the endosperm of ginkgo (Ginkgo biloba) seeds contains ginkbilobin-2 (Gnk2), a novel antifungal protein (Sawano et al., 2007). Ginkgo is one of the oldest gymnosperm species and is sometimes called a 'living fossil'. Gnk2 consists of 108 amino acids with three disulfide bonds and inhibits the growth of plant and human pathogenic fungi such as Fusarium oxysporum and Candida albicans (Sawano et al., 2007).

Interestingly, Gnk2 did not show any sequence similarity to other antimicrobial proteins such as cyclophilin, defensin, miraculin and thaumatin (Sawano et al., 2007). However, this protein does have considerable homology (84-85\%) to embryo-abundant proteins (EAPs) from two other gymnosperms, Picea abies and P. glauca. Plant EAPs are expressed in the late stage of seed maturation and are involved in protecting the plant against damage from environmental stress, especially drought (Shao et al., 2005). However, Gnk2 showed no sequence homology to angiosperm EAPs, indicating that these proteins have important functions during seed development across the subdivision barrier between gymnosperms and angiosperms. Furthermore, the deduced sequences of plant receptor-like kinases (RLKs) from the angiosperms Oryza sativa and Arabidopsis thaliana are also $28-31 \%$ identical to that of Gnk2 (Sawano et al., 2007).

The plant RLKs play major roles in cellular processes and stress response, including hormonal response, cell differentiation, plant growth and development, self-incompatibility and pathogen recognition (Morris \& Walker, 2003). Among them, the cysteine-rich RLKs (CRKs) are a newly classified member with a C- $X_{8}-\mathrm{C}-X_{2}-\mathrm{C}$ motif and are involved in plant defence responses, being induced by pathogen infection (Czernic et al., 1999). The $\mathrm{C}-X_{8}-\mathrm{C}-X_{2}-\mathrm{C}$ motif is completely conserved in the sequences of both Gnk2 and the two EAPs, indi- 
cating that these cysteine residues are involved in functions such as mediating protein-protein interactions and in a critical step in the activation of many animal receptor kinases upon ligand binding (Hardie, 1999). Therefore, the crystallization and structure determination of Gnk2 would help us to understand the roles of the conserved cysteine-rich region Cys62-Cys71-Cys74.

Here, we report the purification, crystallization and preliminary $\mathrm{X}$-ray analysis of native Gnk2 purified from ginkgo seeds and the selenomethionine derivative of recombinant Gnk2 (SeMet-rGnk2) overexpressed using an Escherichia coli expression system.

\section{Materials and methods}

\subsection{Protein purification}

Gnk2 was extracted from ginkgo nuts and partially purified using a gel-filtration column as described previously (Sawano et al., 2007). SeMet-rGnk2 was overexpressed in the periplasm of E. coli BL21 (DE3) by a coexpression system using pET-26b vector (Novagen) and pDsbABCD1 vector encoding the four Dsb proteins that catalyze the formation and isomerization of disulfide bonds (Kurokawa et al., 2000). The DNA sequence encoding Gnk2 (residues Ala1-Phe108) and a stop codon were inserted into the pET-26b vector between $\mathrm{NcoI}$ and BamHI sites. The expressed protein has an additional Met residue at the $\mathrm{NH}_{2}$-terminal sequence of Gnk2. The cells were cotransformed with the pET-26b and pDsbABCD1 vectors. The transformed cells were cultured at $310 \mathrm{~K}$ in M9 medium containing

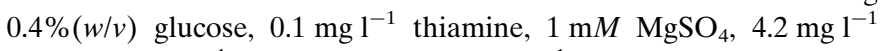
$\mathrm{FeSO}_{4}, 20 \mathrm{mg} \mathrm{l}^{-1}$ kanamycin and $17 \mathrm{mg} \mathrm{l}^{-1}$ chloramphenicol. After 15 min incubation $\left(\mathrm{OD}_{600}=\sim 0.3\right), 50 \mathrm{mg} \mathrm{l}^{-1}$ of Ile, Leu, Val and L-selenomethionine and $100 \mathrm{mg} \mathrm{l}^{-1}$ Lys, Phe and Thr as well as $200 \mathrm{mg} \mathrm{l}^{-1}$ arabinose (for induction of the Dsb proteins) were added to the mixture. The recombinant protein was induced by the addition of $0.5 \mathrm{~m} M$ isopropyl $\beta$-D-thiogalactopyranoside after $15 \mathrm{~min}$ incubation at $310 \mathrm{~K}$. The cells were then cultured for $12 \mathrm{~h}$ at $298 \mathrm{~K}$ and harvested by centrifugation. SeMet-rGnk2 was extracted from the cells by osmotic shock, purified and concentrated using an SPSepharose column (GE Healthcare Bioscience Corp.) equilibrated with $20 \mathrm{~m} M$ MES buffer $\mathrm{pH}$ 6.0. The recombinant protein was eluted with the MES buffer and $300 \mathrm{mM} \mathrm{NaCl}$. For additional purification, the protein solution containing Gnk2 or SeMet-rGnk2 was applied onto the same column. Each protein solution was dialyzed against

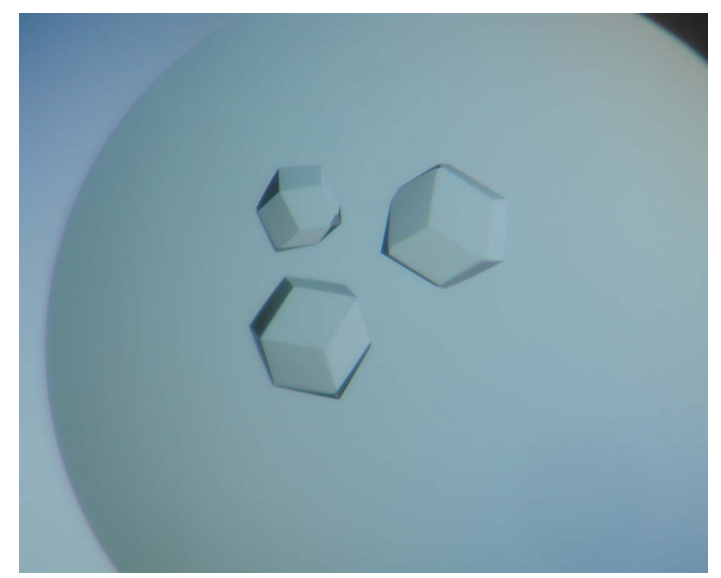

Figure 1

Typical crystals of Gnk2 grown at $293 \mathrm{~K}$ using polyacrylic acid 5100 as the precipitant. The sides of the largest crystal are approximately $0.3 \mathrm{~mm}$. None of the crystals display polarization.
MES buffer and applied onto a Mono-S cation-exchange column (GE Healthcare Bioscience Corp.). The column was eluted with a linear gradient of $0-400 \mathrm{~m} M \mathrm{NaCl}$ in MES buffer. After final purification using a Superdex-75 gel-filtration column (GE Healthcare Bioscience Corp.) equilibrated with MES buffer containing $200 \mathrm{mM} \mathrm{NaCl}$, the protein solution was dialyzed against $10 \mathrm{mM}$ Tris- $\mathrm{HCl} \mathrm{pH} 7.0$ and concentrated to $20 \mathrm{mg} \mathrm{ml}^{-1}$ using VivaSpin (Sartorius AG). The protein concentrations of Gnk2 and SeMet-rGnk2 were estimated from the absorbance at $280 \mathrm{~nm}$ using an absorption coefficient of $6335 \mathrm{M}^{-1} \mathrm{~cm}^{-1}$ (Pace et al., 1995).

\subsection{Dynamic light-scattering study}

Dynamic light-scattering (DLS) measurements were performed using a DynaPro-MSTC instrument (Protein Solutions Inc.). Gnk2 was prepared at $20 \mathrm{mg} \mathrm{ml}^{-1}$ in $10 \mathrm{~m} M$ Tris- $\mathrm{HCl} \mathrm{pH} 7.0$ and measured at $298 \mathrm{~K}$. DLS data were automatically collected using DYNAMICS v.5.1 (Protein Solutions Inc.) and ten data points with baseline levels of $0.998-1.002$ were used to calculate the Stokes radius and the polydispersity of the sample.

\subsection{Crystallization and X-ray data collection}

Initial crystallization tests were carried out with Crystal Screen HT and Index HT kits (Hampton Research) in 96-well plates using the sitting-drop vapour-diffusion method (Corning). For refinement of the crystallization conditions, $1 \mu \mathrm{l}$ protein solution was mixed with an equal volume of reservoir solution and equilibrated against $0.5 \mathrm{ml}$ reservoir solution at $293 \mathrm{~K}$ in 24-well plates using the sitting-drop vapour-diffusion method (Hampton Research). Crystals of Gnk2 and SeMet-rGnk2 suitable for X-ray analysis were obtained under the following reservoir-solution conditions: $21 \%(w / v)$ polyacrylic acid $5100,20 \mathrm{mM} \mathrm{MgCl}_{2}$ and $0.1 M$ MES-NaOH pH 6.6 for Gnk2 and $12 \%(v / v)$ dioxane, $1.6 M$ ammonium sulfate and $0.1 M$ MES-NaOH pH 6.6 for SeMet-rGnk2.

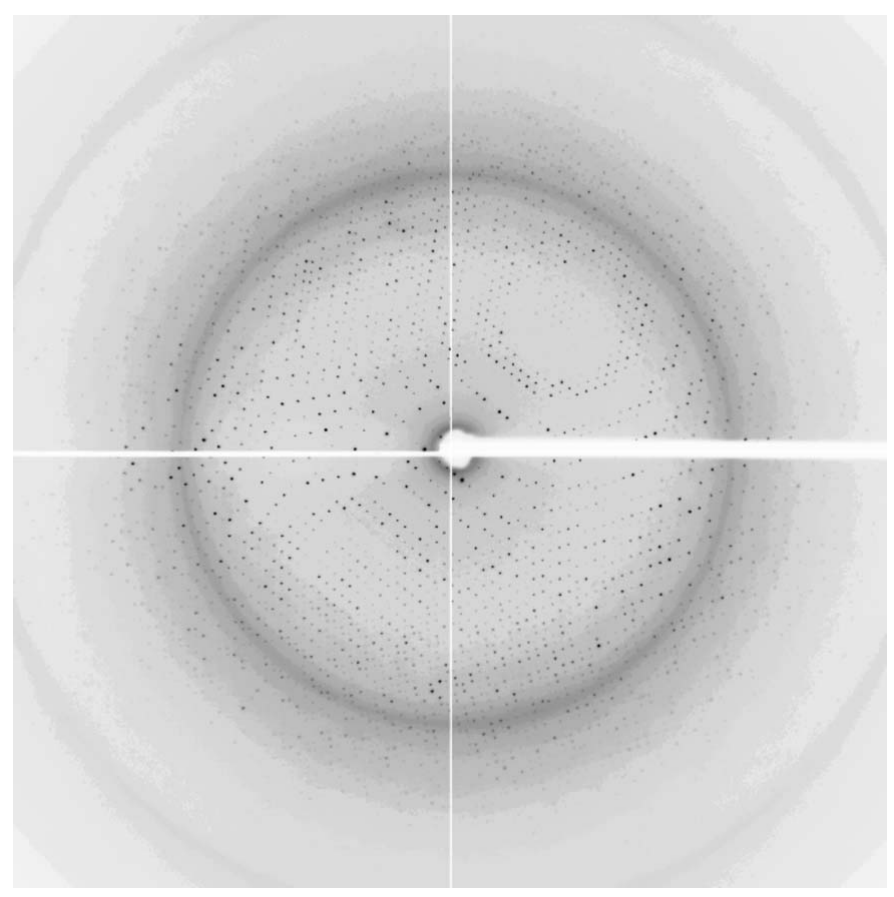

Figure 2

An X-ray diffraction image ( $1.0^{\circ}$ oscillation) from a Gnk2 crystal. The edge of the detector corresponds to a resolution of $2.0 \AA$. 
Table 1

Crystal parameters and data-collection statistics of native ginkbilobin-2 and the selenomethionine derivative of recombinant ginkbilobin-2.

Values in parentheses are for the highest resolution shell.

\begin{tabular}{|c|c|c|c|c|}
\hline & \multirow[b]{2}{*}{ Gnk2 } & \multicolumn{3}{|l|}{ SeMet rGnk2 } \\
\hline & & Peak & Edge & Remote \\
\hline Space group & $P 2_{1} 3$ & \multicolumn{3}{|l|}{$P 2{ }_{1} 3$} \\
\hline Unit-cell parameters $(\AA)$ & $a=b=c=143.2$ & \multicolumn{3}{|c|}{$a=b=c=143.2$} \\
\hline Wavelength $(\AA)$ & 1.00000 & 0.97909 & 0.97934 & 0.96411 \\
\hline Resolution range $(\AA)$ & $\begin{array}{l}50.0-2.38 \\
\quad(2.47-2.38)\end{array}$ & \multicolumn{3}{|c|}{$30.0-2.79(2.89-2.79)$} \\
\hline Observed reflections & 602150 & 272058 & 270119 & 275233 \\
\hline Unique reflections & 39529 & 24549 & 24638 & 24734 \\
\hline Data completeness (\%) & $100(100)$ & $99.8(100)$ & $99.8(100)$ & $99.9(100)$ \\
\hline Redundancy & $15.2(14.5)$ & $11.1(11.0)$ & $11.0(11.0)$ & $11.2(11.1)$ \\
\hline$R_{\text {sym }} \dagger$ & $0.061(0.375)$ & $0.041(0.155)$ & $0.051(0.168)$ & $0.048(0.199)$ \\
\hline$\langle I\rangle /\langle\sigma(I)\rangle$ & $61.2(8.2)$ & $71.7(23.0)$ & $70.9(20.9)$ & $68.2(16.9)$ \\
\hline
\end{tabular}

$\dagger R_{\text {sym }}=\sum_{h k l}\left[\left(\sum_{i}\left|I_{i}-\langle I\rangle\right|\right) / \sum_{i}\left|I_{i}\right|\right]$, where $I_{i}$ is the $i$ th intensity measurement of the reflection $h k l$, including symmetry-related reflections, and $\langle I\rangle$ is its average.

As a cryoprotectant, reservoir solution containing $32 \%(v / v)$ glycerol was added to drops containing protein crystals. The crystals were picked up in a nylon loop (Hampton Research) and mounted for flash-cooling at $100 \mathrm{~K}$. X-ray diffraction data were collected with synchrotron radiation using an ADSC Quantum-210 CCD detector system on the AR-NW12 beamline at Photon Factory (Tsukuba, Japan). The total diffraction data for Gnk2 consisted of 124 images, each exposed for $10 \mathrm{~s}$ with a $1^{\circ}$ oscillation and a wavelength of $1.00000 \AA$ at a crystal-to-detector distance of $235 \mathrm{~mm}$. For multiwavelength anomalous diffraction data collection from the SeMet-

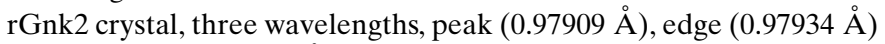
and remote $(0.96411 \AA)$, were determined from the selenium absorption spectrum. The data set at each wavelength was composed of 180 images and collected using a $0.5^{\circ}$ oscillation with a crystal-todetector distance of $291 \mathrm{~mm}$ and an exposure time of $2 \mathrm{~s}$ for each image. All diffraction data were indexed, integrated and scaled with the HKL-2000 program suite (Otwinowski \& Minor, 1997).

\section{Results}

We have established the preparation and crystallization of native and recombinant Gnk2. Although the crystallization conditions for Gnk2 and SeMet-rGnk2 differed, the shapes of their crystals and the time course of the crystallization was quite similar and the crystallographic parameters were identical. The crystals appeared in about $3 \mathrm{~d}$ and grew in a cubic form with all sides approximately $0.3 \mathrm{~mm}$ within two weeks. Fig. 1 shows a typical Gnk2 crystal after two or more weeks. Both crystals belonged to the primitive cubic space group $P 2_{1} 3$, with unit-cell parameters $a=b=c=143.2 \AA$. X-ray diffraction data sets for Gnk2 and SeMet-rGnk2 were processed using data in the resolution ranges $50-2.38 \AA$ and $30-2.79 \AA$, respectively. Fig. 2 shows an image of the X-ray diffraction data of Gnk2. The DLS measurements suggested that the Stokes radius of Gnk2 was $1.68 \mathrm{~nm}$, corresponding to a molecular weight of $10.2 \mathrm{kDa}$ with a polydispersity of $10.8 \%$, and revealed that the native protein is present as a monomer in solution. Assuming the presence of four monomers in an asymmetric unit of the cubic crystal, the typical Matthews coefficient and solvent content were estimated as $5.25 \AA^{3} \mathrm{Da}^{-1}$ and $76.6 \%$, respectively (Matthews, 1968). These values were also consistent with those of the SeMetrGnk2 crystal. The crystal parameters and data-collection statistics are summarized in Table 1. Three-dimensional structure determination will be performed using the multiple-wavelength anomalous dispersion method and the SeMet-rGnk2 data set.

We would like to thank the scientists and staff at the Photon Factory. The synchrotron-radiation experiments were performed at AR-NW12 in the Photon Factory, Tsukuba, Japan (Proposal No. 2006S2-006). We also thank the 'National BioResource Project (NIG, Japan): E. coli' for the gift of JM109 pDsbABCD1 strain. This research was supported in part by the National Project on Protein Structural and Functional Analyses of the Ministry of Education, Culture, Sports, Science and Technology of Japan.

\section{References}

Czernic, P., Visser, B., Sun, W., Savoure, A., Deslandes, L., Marco, Y., Van Montagu, M. \& Verbruggen, N. (1999). Plant J. 18, 321-327.

Hardie, D. G. (1999). Annu. Rev. Plant Physiol. Plant Mol. Biol. 50, 97-131.

Kurokawa, Y., Yanagi, H. \& Yura, T. (2000). Appl. Environ. Microbiol. 66, 3960-3965.

Matthews, B. W. (1968). J. Mol. Biol. 33, 491-497.

Morris, E. R. \& Walker, J. C. (2003). Curr. Opin. Plant Biol. 6, 339-342.

Otwinowski, Z. \& Minor, W. (1997). Methods Enzymol. 276, 307-326.

Pace, C. N., Vajdos, F., Fee, L., Grimsley, G. \& Gray, T. (1995). Protein Sci. 4, 2411-2423.

Sawano, Y., Miyakawa, T., Yamazaki, H., Tanokura, M. \& Hatano, K. (2007). Biol. Chem. 388, 273-280.

Shao, H. B., Liang, Z. S. \& Shao, M. A. (2005). Colloids Surf. B Biointerfaces, 45, 131-135.

Selitrennikoff, C. P. (2001). Appl. Environ. Microbiol. 67, 2883-2894.

Wallace, R. J. (2004). Proc. Nutr. Soc. 63, 621-629. 